\title{
FAST COMMUNICATION
}

\section{THE DRIFT-FLUX ASYMPTOTIC LIMIT OF BAROTROPIC TWO-PHASE TWO-PRESSURE MODELS*}

\author{
ANNALISA AMBROSO ${ }^{\dagger}$, CHRISTOPHE CHALONS A $^{\ddagger}$ FRÉDÉRIC COQUEL ${ }^{\S}$, THOMAS \\ GALIÉ $₫$, EDWIGE GODLEWSKI", PIERRE-ARNAUD RAVIART**, AND NICOLAS SEGUIN ${ }^{\dagger \dagger}$
}

\begin{abstract}
We study the asymptotic behavior of the solutions of barotropic two-phase twopressure models, with pressure relaxation, drag force and external forces. Using Chapman-Enskog expansions close to the expected equilibrium, a drift-flux model with a Darcy type closure law is obtained. Also, restricting this closure law to permanent flows (defined as steady flows in some Lagrangian frame), we can obtain a drift-flux model with an algebraic closure law, in the spirit of Zuber-Findlay models. The example of a two-phase flow in a vertical pipe is described.
\end{abstract}

Key words. two-phase flows, drift-flux models, asymptotic limit

AMS subject classifications. 76T10, 35L60, 35C20

\section{Introduction}

The description of two-phase flows is of great importance. Numerous applications need a clever modeling of such flows, in particular in nuclear engineering. Due to the complexity of these flows, some assumptions must be made according to the configurations under study. Various classes of models exist, and a crucial problem is the understanding of the compatibility between all these models $[9,12,4]$. Indeed, the knowledge of these relationships can help us when a coupling between two-phase flow models from different classes must be performed. In [1], for instance, the coupling of two homogeneous two-phase models with different time scales for the mass transfer is investigated. The relationship between these two models enters in the classical frame of relaxation mechanisms [3], and different ways of coupling are proposed.

Here, we investigate the links between two-pressure models, such as the one proposed by Baer and Nunziato [2], and drift-flux models, like the Zuber-Findlay model [14] (see also [8] or [6] for richer models). The main tools we use are Chapman-Enskog expansions and long-time scalings, but this study does not enter in the theory developed in [3]. These tools enable us to understand the different modeling assumptions and asymptotic expansions that are necessary to bridge two-pressure models and driftflux models. A fundamental step is the use of a drift-flux model with a Darcy closure

\footnotetext{
*Received: March 12, 2008; accepted (in revised version): April 2, 2008. Communicated by Shi Jin.

${ }^{\dagger}$ DEN/DANS/DM2S/SFME/LETR CEA-Saclay, F-91191 Gif-sur-Yvette, France (annalisa.ambroso@cea.fr).

${ }^{\ddagger}$ Université Paris Diderot-Paris 7 and UMR 7598, F-75005 Paris, France (chalons@math. jussieu.fr).

§UPMC Univ Paris 06, UMR 7598, Laboratoire J.-L. Lions, F-75005, Paris, France and CNRS, UMR 7598, Laboratoire J.-L. Lions, F-75005, Paris, France (coquel@ann.jussieu.fr).

IDEN/DANS/DM2S/SFME/LETR CEA-Saclay, F-91191 Gif-sur-Yvette, France (thomas.galie@ cea.fr).

II UPMC Univ Paris 06, UMR 7598, Laboratoire J.-L. Lions, F-75005, Paris, France and CNRS, UMR 7598, Laboratoire J.-L. Lions, F-75005, Paris, France (godlewski@ann.jussieu.fr).

** UPMC Univ Paris 06, UMR 7598, Laboratoire J.-L. Lions, F-75005, Paris, France and CNRS, UMR 7598, Laboratoire J.-L. Lions, F-75005, Paris, France (pa@raviart.com).

${ }^{\dagger \dagger}$ UPMC Univ Paris 06, UMR 7598, Laboratoire J.-L. Lions, F-75005, Paris, France and CNRS, UMR 7598, Laboratoire J.-L. Lions, F-75005, Paris, France (seguin@ann.jussieu.fr).
} 
law (see [7] for related computations).

In this note, the main guidelines are sketched. Full details on the asymptotic developments and the numerical schemes are provided in a forthcoming companion paper.

\section{Two-phase two-pressure models and drift-flux models}

Two-phase two-pressure models [2] are governed by the following set of equations, with $\mathbf{u}=\left(\alpha_{2}, \alpha_{1} \rho_{1}, \alpha_{2} \rho_{2}, \alpha_{1} \rho_{1} u_{1}, \alpha_{2} \rho_{2} u_{2}\right)^{T}$ in $d$ space variables $(d=1,2,3)$ :

$$
\begin{aligned}
& \partial_{t} \alpha_{2}+V_{i}(\mathbf{u}) \cdot \nabla_{x} \alpha_{2}=\Theta(\mathbf{u})\left(p_{2}-p_{1}\right), \quad t>0, x \in \mathbb{R}^{d}, \\
& \partial_{t}\left(\alpha_{1} \rho_{1}\right)+\nabla_{x} \cdot\left(\alpha_{1} \rho_{1} u_{1}\right)=-\Gamma(\mathbf{u}), \\
& \partial_{t}\left(\alpha_{2} \rho_{2}\right)+\nabla_{x} \cdot\left(\alpha_{2} \rho_{2} u_{2}\right)=\Gamma(\mathbf{u}) \text {, } \\
& \partial_{t}\left(\alpha_{1} \rho_{1} u_{1}\right)+\nabla_{x} \cdot\left(\alpha_{1} \rho_{1} u_{1} \otimes u_{1}+\alpha_{1} p_{1} \mathbb{I}\right)-P_{i}(\mathbf{u}) \nabla_{x} \alpha_{1} \\
& =\alpha_{1} \rho_{1} f_{1}(\mathbf{u})+\Lambda(\mathbf{u})\left|u_{2}-u_{1}\right|\left(u_{2}-u_{1}\right), \\
& \partial_{t}\left(\alpha_{2} \rho_{2} u_{2}\right)+\nabla_{x} \cdot\left(\alpha_{2} \rho_{2} u_{2} \otimes u_{2}+\alpha_{2} p_{2} \mathbb{I}\right)-P_{i}(\mathbf{u}) \nabla_{x} \alpha_{2} \\
& =\alpha_{2} \rho_{2} f_{2}(\mathbf{u})+\Lambda(\mathbf{u})\left|u_{1}-u_{2}\right|\left(u_{1}-u_{2}\right),
\end{aligned}
$$

where $\alpha_{k}, \rho_{k}, u_{k}$ are the void fraction, the density and the velocity of the phase $k$, where $k=1,2$ and $\alpha_{1}+\alpha_{2}=1$. We assume that solutions to (2.1) belong to the set $\mathbf{u}=\left(\alpha_{2},\left(1-\alpha_{2}\right) \rho_{1}, \alpha_{2} \rho_{2},\left(1-\alpha_{2}\right) \rho_{1} u_{1}, \alpha_{2} \rho_{2} u_{2}\right)^{T} \in \Omega:=(0,1) \times \mathbb{R}_{+}^{*} \times \mathbb{R}_{+}^{*} \times \mathbb{R}^{d} \times$ $\mathbb{R}^{d}$. The pressures $p_{k}$ are defined by the equations of state $p_{k}=\mathscr{P}_{k}\left(\rho_{k}\right)$, where the $\mathscr{P}_{k}$ satisfy classical assumptions for $k=1,2$ :

$$
\begin{gathered}
\mathscr{P}_{k}^{\prime}\left(\rho_{k}\right)>0 \quad \forall \rho_{k}>0, \\
\lim _{\rho_{k} \rightarrow 0} \mathscr{P}_{k}\left(\rho_{k}\right)=0, \quad \lim _{\rho_{k} \rightarrow \infty} \mathscr{P}_{k}\left(\rho_{k}\right)=+\infty .
\end{gathered}
$$

The vectors $f_{1}$ and $f_{2}$ denote the external forces for phases 1 and 2 , while $\Lambda$ and $\Theta$ correspond to positive relaxation functions and the source term $\Gamma$ corresponds to the mass transfer from phase 1 to phase 2. The two functions $V_{i}$ and $P_{i}$, the so-called interfacial velocity and pressure, are convex combinations of, respectively, $u_{1}$ and $u_{2}$, and $p_{1}$ and $p_{2}$ :

$$
\begin{aligned}
& V_{i}(\mathbf{u})=\beta_{V}(\mathbf{u}) u_{1}+\left(1-\beta_{V}(\mathbf{u})\right) u_{2}, \\
& P_{i}(\mathbf{u})=\beta_{P}(\mathbf{u}) p_{1}+\left(1-\beta_{P}(\mathbf{u})\right) p_{2},
\end{aligned}
$$

with $\beta_{V}, \beta_{P} \in[0,1]$. If $d=1$, such systems are strictly hyperbolic over $\Omega$, except when the wave speed $V_{i}$ identifies with another wave speed $u_{k} \pm \sqrt{\mathscr{P}_{k}^{\prime}\left(\rho_{k}\right)}, k=1,2[2,5]$ (the system is hyperbolic but not strictly hyperbolic if $d>1$ due to the multiplicity of the eigenvalues $u_{k} \cdot n$, for any $n \in \mathbb{R}^{d},\|n\| \neq 0$ ).

Let us focus now on drift-flux models. They read:

$$
\begin{aligned}
& \partial_{t} \tilde{\rho}+\nabla_{x} \cdot(\tilde{\rho} \tilde{u})=0, \\
& \partial_{t}(\tilde{\rho} \tilde{Y})+\nabla_{x} \cdot\left(\tilde{\rho} \tilde{u} \tilde{Y}+\tilde{\rho} \tilde{Y}(1-\tilde{Y}) \tilde{u}_{r}\right)=\tilde{\Gamma}(\tilde{\mathbf{v}}), \\
& \partial_{t}(\tilde{\rho} \tilde{u})+\nabla_{x} \cdot\left(\tilde{\rho} \tilde{u} \otimes \tilde{u}+\tilde{p} \mathbb{I}+\tilde{\rho} \tilde{Y}(1-\tilde{Y}) \tilde{u}_{r} \otimes \tilde{u}_{r}\right)=\tilde{\rho}(1-\tilde{Y}) \tilde{f}_{1}+\tilde{\rho} \tilde{Y} \tilde{f}_{2},
\end{aligned}
$$

where $\tilde{\rho}, \tilde{\rho} \tilde{u}$ and $\tilde{Y}$ are the density of the mixture, the momentum vector of the mixture and the mass fraction of phase 2 . Noting $\tilde{\mathbf{v}}=(\tilde{\rho}, \tilde{\rho} \tilde{Y}, \tilde{\rho} \tilde{u})^{T}$, we define the set of admissible states $\Omega_{D}:=\left\{\tilde{\mathbf{v}} \in \mathbb{R}^{2+d} \mid \tilde{\rho}>0,(\tilde{\rho} \tilde{Y} / \tilde{\rho}) \in(0,1)\right\}$. The pressure $\tilde{p}$ is given 
by some closure law $\tilde{p}=\tilde{\mathscr{P}}(\tilde{\mathbf{v}})$. More precisely, let two classical pressure laws $\tilde{\mathscr{P}}_{1}$ and $\tilde{\mathscr{P}}_{2}$ be given satisfying

$$
\begin{gathered}
\tilde{\mathscr{P}}_{k}^{\prime}\left(\tilde{\rho}_{k}\right)>0, \quad \forall \tilde{\rho}_{k}>0, \\
\lim _{\tilde{\rho}_{k} \rightarrow 0} \tilde{\mathscr{P}}_{k}\left(\tilde{\rho}_{k}\right)=0, \quad \tilde{\tilde{\rho}}_{k \rightarrow \infty} \tilde{\mathscr{P}}_{k}\left(\tilde{\rho}_{k}\right)=+\infty .
\end{gathered}
$$

Then the pressure law $\tilde{p}=\tilde{\mathscr{P}}(\tilde{\mathbf{v}})$ is obtained by solving the $2 \times 2$ nonlinear system

$$
\begin{gathered}
\tilde{p}=\tilde{\mathscr{P}}_{1}(\tilde{\rho}(1-\tilde{Y}) /(1-\tilde{\alpha})), \\
\tilde{\mathscr{P}}_{1}(\tilde{\rho}(1-\tilde{Y}) /(1-\tilde{\alpha}))=\tilde{\mathscr{P}}_{2}(\tilde{\rho} \tilde{Y} / \tilde{\alpha}),
\end{gathered}
$$

where the unknown $(\tilde{p}, \tilde{\alpha})$ belongs to $(0,+\infty) \times(0,1)$. The existence and uniqueness of the solution $(\tilde{p}, \tilde{\alpha})$ is ensured by assumptions (2.4), provided that $\tilde{\mathbf{v}} \in \Omega_{D}$. The relative velocity vector $\tilde{u}_{r}$ corresponds to the difference between the velocity of phase 2 and the velocity of phase 1 and is given by the closure law $\tilde{u}_{r}=\tilde{\Phi}(\tilde{\mathbf{v}})$ (see [9] and [10] for explicit laws). The precise form of the vector-valued function $\tilde{\Phi}$ will be discussed in Section 4. The functions $\tilde{\Gamma}, \tilde{f}_{1}$ and $\tilde{f}_{2}$ denote the mass transfer and the external forces.

\section{Asymptotic analysis}

The goal of this section is to obtain a model similar to (2.3) using asymptotic arguments, starting from a two-phase two-pressure model (2.1).

3.1. The time scales of the source terms. In order to study the asymptotic limits of (2.1), we must make explicit the different scales of the system. To this aim, we use a small parameter $\varepsilon$ which describes the strength of perturbations of a dimensionless one-velocity one-pressure equilibrium flow. The first perturbation we are interested in concerns the difference of the velocities. In order to obtain a first order in $\varepsilon$ perturbation, we set

$$
\Lambda(\mathbf{u})=\frac{\lambda(\mathbf{u})}{\varepsilon^{2}}
$$

since the drag force is quadratic with respect to $u_{1}-u_{2}$. It is classical to assume that the pressure relaxation is much faster that the relaxation due to the drag force [9]. Therefore, we use the following scaling for the pressure relaxation coefficient:

$$
\Theta(\mathbf{u})=\frac{\theta(\mathbf{u})}{\varepsilon^{2}}
$$

(the pressure relaxation term is linear with respect to $p_{1}-p_{2}$ ). Besides, we assume that the characteristic time associated with the mass transfer is much larger than the characteristic times of the pressure relaxation and of the drag effects. We then use the scaling

$$
\Gamma(\mathbf{u})=\varepsilon \gamma(\mathbf{u})
$$

Letting $\Gamma$ be linear with respect to $\varepsilon$ will enable us to preserve the mass transfer term when we investigate the long-time behavior of the solutions in Section 3.3. 


\subsection{First order equilibrium: a drift-flux model with a Darcy law.}

This first step in bridging models (2.1) and (2.3) lies in the study of the first order asymptotic limit of $(2.1)$.

Solutions to (2.1) with (3.1-3.2) are now parameterized by $\varepsilon$, and we denote them by $\mathbf{u}^{\varepsilon}$. Let us introduce some new variables:

$$
\begin{aligned}
\rho^{\varepsilon} & =\alpha_{1}^{\varepsilon} \rho_{1}^{\varepsilon}+\alpha_{2}^{\varepsilon} \rho_{2}^{\varepsilon}, & \rho^{\varepsilon} u^{\varepsilon} & =\alpha_{1}^{\varepsilon} \rho_{1}^{\varepsilon} u_{1}^{\varepsilon}+\alpha_{2}^{\varepsilon} \rho_{2}^{\varepsilon} u_{2}^{\varepsilon}, & \rho^{\varepsilon} Y^{\varepsilon} & =\alpha_{2}^{\varepsilon} \rho_{2}^{\varepsilon}, \\
u_{r}^{\varepsilon} & =u_{2}^{\varepsilon}-u_{1}^{\varepsilon}, & p^{\varepsilon} & =\alpha_{1}^{\varepsilon} p_{1}^{\varepsilon}+\alpha_{2}^{\varepsilon} p_{2}^{\varepsilon}, & p_{r}^{\varepsilon} & =p_{2}^{\varepsilon}-p_{1}^{\varepsilon} .
\end{aligned}
$$

By easy calculations, one can deduce from (2.1) the following equations:

$$
\begin{aligned}
& \partial_{t} \rho^{\varepsilon}+\nabla_{x} \cdot\left(\rho^{\varepsilon} u^{\varepsilon}\right)=0, \\
& \partial_{t}\left(\rho^{\varepsilon} Y^{\varepsilon}\right)+\nabla_{x} \cdot\left(\rho^{\varepsilon} u^{\varepsilon} Y^{\varepsilon}+\rho^{\varepsilon} Y^{\varepsilon}\left(1-Y^{\varepsilon}\right) u_{r}^{\varepsilon}\right)=\Gamma^{\varepsilon}, \\
& \partial_{t}\left(\rho^{\varepsilon} u^{\varepsilon}\right)+\nabla_{x} \cdot\left(\rho^{\varepsilon} u^{\varepsilon} \otimes u^{\varepsilon}+p^{\varepsilon} \mathbb{I}+\rho^{\varepsilon} Y^{\varepsilon}\left(1-Y^{\varepsilon}\right) u_{r}^{\varepsilon} \otimes u_{r}^{\varepsilon}\right) \\
& \quad=\rho^{\varepsilon}\left(1-Y^{\varepsilon}\right) f_{1}^{\varepsilon}+\rho^{\varepsilon} Y^{\varepsilon} f_{2}^{\varepsilon}, \\
& \partial_{t} u_{r}^{\varepsilon}+u_{r}^{\varepsilon} \cdot \nabla_{x} u^{\varepsilon}+u^{\varepsilon} \cdot \nabla_{x} u_{r}^{\varepsilon}+\nabla_{x} \cdot\left(\left(1 / 2-Y^{\varepsilon}\right) u_{r}^{\varepsilon} \otimes u_{r}^{\varepsilon}\right)+\left(1 / \rho_{2}^{\varepsilon}-1 / \rho_{1}^{\varepsilon}\right) \nabla_{x} p^{\varepsilon} \\
& \quad+\left(\beta_{P} /\left(\rho^{\varepsilon} Y^{\varepsilon}\right)-\left(1-\beta_{P}\right) /\left(\rho^{\varepsilon}\left(1-Y^{\varepsilon}\right)\right)+1 / \rho_{1}^{\varepsilon}-1 / \rho_{2}^{\varepsilon}\right) p_{r}^{\varepsilon} \nabla_{x} \alpha_{2}^{\varepsilon} \\
& \quad+\rho^{\varepsilon} /\left(\rho_{1}^{\varepsilon} \rho_{2}^{\varepsilon}\right) \nabla_{x} p_{r}^{\varepsilon}=f_{2}^{\varepsilon}-f_{1}^{\varepsilon}-\lambda^{\varepsilon}\left|u_{r}^{\varepsilon}\right| u_{r}^{\varepsilon} /\left(\varepsilon^{2} \rho^{\varepsilon} Y^{\varepsilon}\left(1-Y^{\varepsilon}\right)\right), \\
& \partial_{t} p_{r}^{\varepsilon}+u_{r}^{\varepsilon} \cdot \nabla_{x} p^{\varepsilon}+\left(\rho_{2}^{\varepsilon} \mathscr{P}_{2}^{\prime}\left(\rho_{2}^{\varepsilon}\right)-\rho_{1}^{\varepsilon} \mathscr{P}_{1}^{\prime}\left(\rho_{1}^{\varepsilon}\right)\right)\left(\nabla_{x} \cdot u^{\varepsilon}-u_{r}^{\varepsilon} \cdot \nabla_{x} Y^{\varepsilon}\right) \\
& \quad+\left(Y^{\varepsilon} \rho_{1}^{\varepsilon} \mathscr{P}_{1}^{\prime}\left(\rho_{1}^{\varepsilon}\right)+\left(1-Y^{\varepsilon}\right) \rho_{2}^{\varepsilon} \mathscr{P}_{2}^{\prime}\left(\rho_{2}^{\varepsilon}\right)\right) \nabla_{x} \cdot u_{r}^{\varepsilon}+\left(\alpha_{1}^{\varepsilon} u_{2}^{\varepsilon}-\alpha_{2}^{\varepsilon} u_{1}^{\varepsilon}\right) \cdot \nabla_{x} p_{r}^{\varepsilon} \\
& \quad+\left(\beta_{V} \rho_{2}^{\varepsilon} \mathscr{P}_{2}^{\prime}\left(\rho_{2}^{\varepsilon}\right) / \alpha_{2}^{\varepsilon}-\left(1-\beta_{V}\right) \rho_{1}^{\varepsilon} \mathscr{P}_{1}^{\prime}\left(\rho_{1}^{\varepsilon}\right) / \alpha_{1}^{\varepsilon}-p_{r}^{\varepsilon}\right) u_{r}^{\varepsilon} \cdot \nabla_{x} \alpha_{2}^{\varepsilon} \\
& \quad=-\left(\rho_{1}^{\varepsilon} \mathscr{P}_{1}^{\prime}\left(\rho_{1}^{\varepsilon}\right) / \alpha_{1}^{\varepsilon}+\rho_{2}^{\varepsilon} \mathscr{P}_{2}^{\prime}\left(\rho_{2}^{\varepsilon}\right) / \alpha_{2}^{\varepsilon}\right) \theta^{\varepsilon} p_{r}^{\varepsilon} / \varepsilon^{2} .
\end{aligned}
$$

It is worth noticing that under assumptions (2.2), the application which maps $\mathbf{u}^{\varepsilon} \in \Omega$ to $\left(\rho^{\varepsilon}, \rho^{\varepsilon} Y^{\varepsilon}, \rho^{\varepsilon} u^{\varepsilon}, u_{r}^{\varepsilon}, p_{r}^{\varepsilon}\right)^{T}$ is a diffeomorphism. Therefore, $\alpha_{2}^{\varepsilon}, \rho_{1}^{\varepsilon}, \rho_{2}^{\varepsilon}, f_{1}^{\varepsilon}, f_{2}^{\varepsilon}, \ldots$ must be understood in (3.4) as functions of $\left(\rho^{\varepsilon}, \rho^{\varepsilon} Y^{\varepsilon}, \rho^{\varepsilon} u^{\varepsilon}, u_{r}^{\varepsilon}, p_{r}^{\varepsilon}\right)^{T}$ (we keep for simplicity the same notations whatever the dependence of the functions on $\mathbf{u}^{\varepsilon}$ or on $\left(\rho^{\varepsilon}, \rho^{\varepsilon} Y^{\varepsilon}, \rho^{\varepsilon} u^{\varepsilon}, u_{r}^{\varepsilon}, p_{r}^{\varepsilon}\right)^{T}$ is). We thus deduce that the system (3.4) is closed.

We are interested in studying solutions of (3.4) near the equilibrium defined by

$$
u_{r}^{\varepsilon}=0 \text { and } p_{r}^{\varepsilon}=0 \text {, }
$$

and in obtaining a closed system which describes such solutions up to the first order in $\varepsilon$. With this in mind, we follow the Chapman-Enskog method and focus on solutions of the form

$$
\begin{aligned}
& u_{r}^{\varepsilon}=\varepsilon u_{r 1}^{\varepsilon}+\mathcal{O}\left(\varepsilon^{2}\right), \\
& p_{r}^{\varepsilon}=\varepsilon p_{r 1}^{\varepsilon}+\mathcal{O}\left(\varepsilon^{2}\right) .
\end{aligned}
$$

These expansions and the last two equations of (3.4) lead us to define

$$
\begin{aligned}
\left|u_{r 1}^{\varepsilon}\right| u_{r 1}^{\varepsilon} & =\frac{\rho^{\varepsilon} Y^{\varepsilon}\left(1-Y^{\varepsilon}\right)}{\lambda^{\varepsilon}}\left(f_{2}^{\varepsilon}-f_{1}^{\varepsilon}+\left(\frac{1}{\rho_{1}^{\varepsilon}}-\frac{1}{\rho_{2}^{\varepsilon}}\right) \nabla_{x} p^{\varepsilon}\right), \\
p_{r 1}^{\varepsilon} & =0 .
\end{aligned}
$$

We are now in position to propose a first order equilibrium system associated with the two-phase two-pressure model (2.1), i.e., a reduced model in which only second (and higher) order terms in $\varepsilon$ have been neglected. 
Proposition 3.1. Let us denote by $\mathbf{v}=(\rho, \rho Y, \rho u)^{T} \in \Omega_{D}$ and assume that $\Gamma, \lambda, f_{1}$ and $f_{2}$ only depend on $\mathbf{v}$. Consider the system

$$
\begin{aligned}
& \partial_{t} \rho+\nabla_{x} \cdot(\rho u)=0 \\
& \partial_{t}(\rho Y)+\nabla_{x} \cdot\left(\rho u Y+\rho Y(1-Y) u_{r}\right)=\Gamma(\mathbf{v}) \\
& \partial_{t}(\rho u)+\nabla_{x} \cdot\left(\rho u \otimes u+p \mathbb{I}+\rho Y(1-Y) u_{r} \otimes u_{r}\right) \\
& =\rho(1-Y) f_{1}(\mathbf{v})+\rho Y f_{2}(\mathbf{v})
\end{aligned}
$$

where

$$
\left|u_{r}\right| u_{r}=\varepsilon^{2} \frac{\rho Y(1-Y)}{\lambda(\mathbf{v})}\left(f_{2}(\mathbf{v})-f_{1}(\mathbf{v})+\left(\frac{1}{\rho_{1}(\mathbf{v})}-\frac{1}{\rho_{2}(\mathbf{v})}\right) \nabla_{x} p\right),
$$

and with the pressure law $p=\mathscr{P}(\mathbf{v})$, given by the solution $\left(p, \alpha_{2}^{e}\right)$ of the $2 \times 2$ nonlinear system

$$
\begin{aligned}
& p=\mathscr{P}_{1}\left(\rho(1-Y) /\left(1-\alpha_{2}^{e}\right)\right), \\
& \mathscr{P}_{1}\left(\rho(1-Y) /\left(1-\alpha_{2}^{e}\right)\right)=\mathscr{P}_{2}\left(\rho Y / \alpha_{2}^{e}\right) .
\end{aligned}
$$

The system (3.8-3.10) is closed and its solutions correspond to equilibrium solutions of the two-phase two-pressure model (2.1) up to the first order in $\varepsilon$.

In order to prove that this system is closed, we only need to study the system (3.10). As soon as $\mathbf{v} \in \Omega_{D}$, simple computations allow to check that assumptions (2.2) on the pressure laws ensure that $\alpha_{2}^{e} \in(0,1)$ and that there exists a function $\mathscr{P}$ that maps $\mathbf{v}$ to $p$ (see (2.4) and (2.5) for a similar discussion). Note that these assumptions also ensure that the pressure laws are invertible, so that the functions $\rho_{k}(\mathbf{v}):=\mathscr{P}_{k}^{-1}(\mathscr{P}(\mathbf{v})), \mathbf{v} \in \Omega_{D}$ in (3.9) make sense.

Besides, only terms of order $\varepsilon^{2}$ and higher have been neglected in (3.8-3.10), thanks to the assumptions on $\lambda, \Gamma, f_{1}$ and $f_{2}$ made in Proposition 3.1. The term $u_{r} \otimes u_{r}$, of order $\varepsilon^{2}$, has been kept in (3.8) in agreement with the classical form of drift-flux models. It is also important to remark that, due to the order in $\varepsilon^{2}$ of the pressure relaxation, the first order pressure correction $p_{r 1}^{\varepsilon}$ is null, and consequently the system (3.8-3.10) is independent of $\theta$.

This system enables one to describe flows near the equilibrium (3.5) of system (2.1) (see [7] for a study of a related system). It is the first step towards a classical drift-flux model. Indeed, $\mathbf{v}=(\rho, \rho Y, \rho u)^{T}$ in $(3.8)$ and $\tilde{\mathbf{v}}=(\tilde{\rho}, \tilde{\rho} \tilde{Y}, \rho \tilde{u})^{T}$ in $(2.3)$ verify the same set of partial differential equations, with $\Gamma \equiv \tilde{\Gamma}, f_{1} \equiv \tilde{f}_{1}$ and $f_{2} \equiv \tilde{f}_{2}$, the only difference being that the hydrodynamic closure law (3.9) is a Darcy-like law, in the sense that $u_{r}$ depends not only on $\mathbf{v}$ but also on its derivative via $\nabla_{x} p$.

3.3. Permanent flows: from a Darcy law to a zeroth order law. In order to obtain a zeroth order hydrodynamic closure law, we investigate permanent flows. We define such flows as the long-time limit of the solutions of (3.8) along the characteristics of the flow. We then focus on the characteristics of the flow and rewrite system (3.8) in the associated frame of reference. Let us consider the differential system

$$
\left\{\begin{array}{l}
\frac{d x}{d t}=u(x, t), \quad t>0 \\
x(0)=\xi
\end{array}\right.
$$


where $\xi \in \mathbb{R}^{d}$. We denote by $x(\xi, t)$ the solution of (3.11) and introduce the associated change of coordinates

$$
\bar{\phi}(\xi, t)=\phi(x(\xi, t), t),
$$

where $\phi$ is a given function in Eulerian coordinates and $\bar{\phi}$ the same function in Lagrangian coordinates. We then obtain

$$
\partial_{t} \bar{\phi}=\overline{\partial_{t} \phi+u \cdot \nabla_{x} \phi}
$$

The equations of (3.8) becomes in the new frame of reference

$$
\begin{aligned}
& \partial_{t} \bar{\rho}+\overline{\rho \nabla_{x} \cdot u}=0, \\
& \partial_{t} \overline{\rho Y}+\overline{\rho Y \nabla_{x} \cdot u}+\overline{\nabla_{x} \cdot\left(\rho Y(1-Y) u_{r}\right)}=\varepsilon \bar{\gamma}, \\
& \partial_{t} \overline{\rho u}+\overline{\left(\rho \nabla_{x} \cdot u\right) u}+\overline{\nabla_{x} p}+\overline{\nabla_{x} \cdot\left(\rho Y(1-Y) u_{r} \otimes u_{r}\right)}=\overline{\rho\left((1-Y) f_{1}+Y f_{2}\right)} .
\end{aligned}
$$

In order to investigate the long-time limit, we use the scaling $s=\varepsilon t$. This leads to

$$
\begin{aligned}
& \varepsilon \partial_{s} \bar{\rho}+\overline{\rho \nabla_{x} \cdot u}=0, \\
& \varepsilon \partial_{s} \overline{\rho Y}+\overline{\rho Y \nabla_{x} \cdot u}+\overline{\nabla_{x} \cdot\left(\rho Y(1-Y) u_{r}\right)}=\varepsilon \bar{\gamma}, \\
& \varepsilon \partial_{s} \overline{\rho u}+\overline{\left(\rho \nabla_{x} \cdot u\right) u}+\overline{\nabla_{x} p}+\overline{\nabla_{x} \cdot\left(\rho Y(1-Y) u_{r} \otimes u_{r}\right)}=\overline{\rho\left((1-Y) f_{1}+Y f_{2}\right)} .
\end{aligned}
$$

At the zeroth order, what remains is

$$
\begin{aligned}
& \overline{\rho \nabla_{x} \cdot u}=0, \\
& \partial_{s} \overline{\rho Y}+\overline{\nabla_{x} \cdot\left(\rho Y(1-Y) u_{r 1}\right)}=\bar{\gamma}, \\
& \overline{\nabla_{x} p}=\overline{\rho\left((1-Y) f_{1}+Y f_{2}\right)} .
\end{aligned}
$$

Assuming now that the flow governed by (3.8) satisfies this relation for all $t>0$ and $x \in \mathbb{R}^{d}$, the closure law (3.9) becomes

$$
\begin{aligned}
\left|u_{r}\right| u_{r}=\varepsilon^{2} \frac{\rho Y(1-Y)}{\lambda(\mathbf{v})}\left(f_{2}(\mathbf{v})\right. & -f_{1}(\mathbf{v}) \\
& \left.+\left(\frac{1}{\rho_{1}(\mathbf{v})}-\frac{1}{\rho_{2}(\mathbf{v})}\right)\left(\rho(1-Y) f_{1}(\mathbf{v})+\rho Y f_{2}(\mathbf{v})\right)\right),
\end{aligned}
$$

from which one can deduce a zeroth order closure law $u_{r}=\Phi(\mathbf{v})$.

REMARK 3.2. Provided that $\lambda, f_{1}, f_{2}$ and $\mathscr{P}$ are only functions of $\rho$ and $\rho Y$, equations (3.15), (3.16) with the closure laws

$$
\bar{p}=\mathscr{P}(\overline{\mathbf{v}}) \quad \text { and } \quad \overline{\left|u_{r 1}\right| u_{r 1}}=\overline{\frac{\rho Y(1-Y)}{\lambda}\left(f_{2}-f_{1}+\left(\frac{1}{\rho_{1}}-\frac{1}{\rho_{2}}\right) \nabla_{x} p\right)}
$$

constitute a closed system very similar to those which govern the evolution of the saturation of a fluid in a porous medium, coupled with a Darcy law.

REMARK 3.3. One can here notice the importance of the scaling $\Gamma=\varepsilon \gamma$. Indeed, when $\varepsilon \rightarrow 0$, the second equation of (3.13) directly vanishes, whereas, if $\Gamma$ had been independent of $\varepsilon$, the limit $\varepsilon \rightarrow 0$ would yield $\Gamma \equiv 0$. 


\section{The asymptotic drift-flux model}

We have obtained a closed drift-flux model, composed of the PDE's (3.8) with the zeroth order hydrodynamic closure law (3.17) and the pressure law given by the solution of (3.10). This last equation is exactly the pressure law $\tilde{\mathscr{P}}$ which appears for classical drift-flux models (2.3), provided that $\mathscr{P}_{k} \equiv \tilde{\mathscr{P}}_{k}, k=1,2$. Moreover, $\mathbf{v}=$ $(\rho, \rho Y, \rho u)$ in (3.8) and $\tilde{\mathbf{v}}=(\tilde{\rho}, \tilde{\rho} \tilde{Y}, \rho \tilde{u})$ in (2.3) verify the same set of PDE's, with $f_{1} \equiv \tilde{f}_{1}, f_{2} \equiv \tilde{f}_{2}$ and $\Gamma \equiv \tilde{\Gamma}$. Then, it remains to address the hydrodynamic law (3.17).

4.1. The case of a vertical pipe with buoyancy effects. In order to understand the connection between the hydrodynamic laws $\Phi$ and $\tilde{\Phi}$, we present the case of a dispersed flow in a vertical confined channel only subject to the gravity force, that is to say, we only consider classical buoyancy effects. The model is now one-dimensional, $x$ is the upward direction, and thus $f_{1}=f_{2}=-g$, where $g$ is the gravity constant. Assume that phase 1 is the air and phase 2 is the liquid; then $\rho_{1} \ll \rho_{2}$.

Following [10], the drag force in the two-pressure model (2.1) can be defined by

$$
\Lambda(\mathbf{u})\left|u_{r}\right| u_{r}=\frac{3}{8 R_{D}} \alpha_{1} \rho_{2} C_{D}(\mathbf{u})\left|u_{r}\right| u_{r}
$$

where $R_{D}$ is the bubble radius (assumed to be constant) and $C_{D}$ is the drag coefficient. For the present configuration of flow, Ishii and Zuber proposed the following formula in [11]:

$$
C_{D}(\mathbf{u})=\frac{4 R_{D}}{3}\left(\alpha_{2}\right)^{-\vartheta} \sqrt{\left(\rho_{2}-\rho_{1}\right) g / \sigma}
$$

where $\sigma$ denotes the surface tension, and the value of the constant $\vartheta$ is given according to the ratio of the dynamical viscosities of the phases. This leads to

$$
\Lambda(\mathbf{u})\left|u_{r}\right| u_{r}=\frac{1}{2} \alpha_{1}\left(\alpha_{2}\right)^{-\vartheta} \rho_{2} \sqrt{\left(\rho_{2}-\rho_{1}\right) g / \sigma}\left|u_{r}\right| u_{r}
$$

from which we can deduce $\Lambda$ (note that $\Lambda$ can also be expressed as a function of $\mathbf{v}$ ). On the other hand, Equation (3.17) reduces to

$$
\Lambda(\mathbf{u})\left|u_{r}\right| u_{r}=-\alpha_{1} \alpha_{2}\left(\rho_{2}-\rho_{1}\right) g
$$

Due to the configuration of the flow, both velocities $u_{1}$ and $u_{2}$ are negative and $u_{2} \leqslant u_{1}$, which yields $\Lambda(\mathbf{u}) \geqslant 0$. Identifying the two latter equations enables us to deduce the following form for the relative velocity:

$$
u_{r}=-\sqrt{2}\left(\frac{\sigma\left(\rho_{2}-\rho_{1}\right) g}{\left(\rho_{2}\right)^{2}}\right)^{1 / 4}\left(\alpha_{2}\right)^{(1+\vartheta) / 2} .
$$

This definition corresponds to a classical hydrodynamic closure law $\tilde{u}_{r}=\tilde{\Phi}(\tilde{\mathbf{v}})$ for drift-flux models (see for instance [8] or [6] for such models and extensions). Actually, this correspondence is not so surprising, since it is a classical way of modeling in twophase flows. The cornerstone of this derivation in modeling of two-phase flows is the balance of the gradient of pressure with the external forces, which is recovered here by the study of permanent flows. 
4.2. Some remarks on the derivation. Let us first provide a comment on the entropy criterion. The functions $V_{i}$ and $P_{i}$ are only assumed to be convex combinations of the velocities and pressures respectively. For a more detailed analysis regarding the consistency of two-phase two-pressure models with the entropy principle, additional requirements on these two quantities must be added [5]. This should enable us to study the dissipative structure of the relaxation terms and the nonlinear stability of the asymptotic limits proposed here, following [3].

On the other hand, a theoretical study of the asymptotic limits described in this paper should be performed. In particular, the works of Yong [13] on the relation of the dissipative structure of the relaxation phenomenon with the convergence towards equilibrium smooth flows could be very useful to justify the asymptotic link between the two-pressure model (2.1) and the drift-flux model (2.3).

In general, system (3.8) is directly obtained from (2.1) by algebraic manipulations and restricting to one-pressure flows, while the drift law is derived using the balance of the buoyancy effects by the drag forces. The novelty of our work is the proposition of a hierarchy between two-pressure models and drift-flux models by the use of asymptotic mechanisms.

Acknowledgement. The authors would like to thank Olivier Grégoire (CEA Saclay) for valuable insights in the origins of drift-flux models and illuminating discussions. This work falls within the scope of a joint research program on the coupling of multiphase flow models between CEA Saclay and Laboratoire Jacques-Louis Lions (http://www.ann.jussieu.fr/groupes/cea). It was partially supported by the Neptune Project, funded by CEA (Commissariat à l'Énergie Atomique), EDF (Électricité de France), IRSN (Institut de Radioprotection et de Sûreté Nucléaire) and AREVA-NP.

\section{REFERENCES}

[1] A. Ambroso, C. Chalons, F. Coquel, E. Godlewski, F. Lagoutière, P.A. Raviart and N. Seguin, The coupling of homogeneous models for two-phase flows, Int. J. Finite Volumes, 4(1), $1-39,2007$.

[2] M.R. Baer and J.W. Nunziato, A two-phase mixture theory for the deflagration-to-detonation transition (DDT) in reactive granular materials, Int. J. Multiphase Flow, 12, 861-889, 1986.

[3] G.Q. Chen, C.D. Levermore and T.P. Liu, Hyperbolic conservation laws with stiff relaxation terms and entropy, Comm. Pure Appl. Math., 47(6), 787-830, 1994.

[4] D.A. Drew and S. Passman, Theory of Multicomponent Fluids, Springer, New-York, 1998.

[5] T. Gallouët, J.M. Hérard and N. Seguin, Numerical modeling of two-phase flows using the two-fluid two-pressure approach, Math. Models Methods Appl. Sci., 14(5), 663-700, 2004.

[6] O. Grégoire and M. Martin, Derivation of a well-posed and multidimensional drift-flux model for boiling flows, C.R. Mecanique, 333(6), 459-466, 2005.

[7] H. Guillard and F. Duval, A Darcy law for the drift velocity in a two-phase flow model, J. Comput. Phys., 224(1), 288-313, 2007.

[8] T. Hibiki and M. Ishii, One-dimensional drift-flux model and constitutive equations for relative motion between phases in various two-phase flow regimes, Int. J. of Heat and Mass Transfer, 46, 4935-4948, 2003.

[9] M. Ishii, Thermo-Fluid Dynamic Theory of Two-Phase Flow, Eyrolles, Paris, 1975.

[10] M. Ishii and K. Mishima, Two-fluid model and hydrodynamic constitutive relations, Nuclear Engrg. Design, 82(2-3), 107-126, 1984.

[11] M. Ishii and N. Zuber, Drag coefficient and relative velocity in bubbly, droplet or particulate flows, AIChE J., 25, 843-855, 1979.

[12] H.B. Stewart and B. Wendroff, Two-phase flow: models and methods, J. Comput. Phys., 56(3), 363-409, 1984. 
[13] W.A. Yong, Entropy and global existence for hyperbolic balance laws, Arch. Ration. Mech. Anal., 172(2), 247-266, 2004.

[14] N. Zuber and J.A. Findlay, Average volumetric concentration in two-phase flow systems, J. Heat Transfer, 68, 453-468, 1965. 